\title{
Mobilität und Migration im Mittelmeerraum Zur mythotopographischen Erzähl- und Erinnerungskultur der Griechen am Beispiel der Familie der Europa
}

Von der Antike bis heute ist der Mythos der von Zeus aus Phönizien entführten Königstochter Europa kontinuierlich mit dem gleichnamigen Erdteil in Verbindung gebracht worden. Zumal in der Neuzeit, in der Moderne und in der Gegenwart, wurde und wird die Königstochter als Gründungsheroin Europas imaginiert und, auf der Suche nach den Ursprüngen und einer Identität Europas, als Symbol für den Kontinent bzw. die Gemeinschaft der Europäer gehandelt. ${ }^{I}$ Herodots bereits im vierten Jahrhundert v. Chr. ausdrücklich und nachvollziehbar geäußerte Ansicht in seinen Historien (Hdt. 4,45,I-4), ${ }^{2}$ die Benennung des Kontinents stehe mit dem Entführungsmythos nicht in einem ursächlichen Zusammenhang, hat Denker, Literaten und Künstler in und seit der Antike nicht von Verknüpfungen und Verschmelzungen der mit dem Europa abgehalten. Dies ist umso verwunderlicher, als wir tatsächlich keinerlei sicheren Anhalt für einen ursächlichen Zusammenhang haben, zumal keinen etymologisch begründeten. Es gab im Altertum eine Vielzahl sowohl mythischer und kultisch verehrter Gestalten als auch geographischer Angaben mit dem Namen „Europa“, und die Etymologie des Wortes ist höchst umstritten. In der Frage, ob der Name griechischen Ursprungs sei, haben moderne Sprachwissenschaftler bis heute zu keinem Konsens gefunden. ${ }^{3}$ Europa ist derzeitigem Forschungsstand nach ein Wort aus einer unbekannten Sprache, mit unbekannter Bedeutung. Auf verschlungenen

I Vgl. dazu Renger/Ißler 2009, 63-99.

2 Ausgaben und Übersetzungen antiker Texte werden im Folgenden sowie in der Bibliographie im Anhang nicht gesondert angeführt. Autorennamen und Werktitel werden im Argumentationsgang ausgeschrieben, bei Stellenangaben aber abgekürzt angegeben, entsprechend den Konventionen in DNP 3 (I997) = Der Neue Pauly. Enzyklopädie der Antike. Hg. von Cancik, Schneider, und Landfester, Altertum Bd. 3 (Cl-Epi), Stuttgart/Weimar I997, XXXVI-XLIVI. Nach Autorennamen- und Werktitelnennung folgen jeweils Angaben wie Buch, Kapitel, Abschnitt, Vers(e) etc. - Für einschlägige Lexika werden Abkürzungen verwendet: LIMC = Lexicon Iconographicum Mythologiae Classicae. Hg. von Boardman u.a. I98Iff.; RAC = Reallexikon für Antike und Christentum. Hg. von Schöllgen u.a. I950ff.; RE = Paulys Real-Encyklopädie der classischen Altertumswissenschaft. Hg. von Wissowa u.a. I893-I980; Roscher = Ausführliches Lexikon der griechischen und römischen Mythologie. Hg. von Roscher I884-I937.

3 Vgl. Musäus 2009, der mit Blick auf die Wortherkunft gegenüber Ableitungen und vor allem Erkenntnismöglichkeiten - wie vor ihm z. B. auch Demandt I998, I40 und Hartmann 2003, 40 - einen skeptischen Standpunkt einnimmt. Etliche Forscher sprechen sich, bei aller Dunkelheit der Quellenlage, gegen einen semitischen und für einen (vor)griechischen Ursprung aus, so z.B. Ninck I945, I9; Bühler I968, 24f., 44; Dombrowsky I984, I-40; Schumacher I990, I2. 
Wegen des Transfers eines Mythos gelangte es zu einer phönizischen Prinzessin, die laut Herodots Historien $(4,45,4)$ niemals das europäische Festland betreten hat.

Trotz der Dunkelheit der Etymologie und der Vielzahl mythischer Europagestalten wurde die von Zeus entführte Europa zu einer europäischen Gedächtnisfigur. Im Zuge ihrer Transformation in literarischen und anderen Medien der Antike wurde die Geschichte der Entführung einer Stilisierung unterzogen, die den Kontinent als performatives Produkt von Bewegung durch den Mittelmeerraum erscheinen ließ: Der Kontinent, so hieß es, habe seinen Namen von der mythischen Figur, die übers Mittelmeer ,nach Europa' gekommen sei.

Zur Bildung und Erhärtung dieser Behauptung trugen m. E. drei Umstände bei, die in den hier angestellten Überlegungen näher betrachtet werden: (I) Unter den zahlreichen einzelnen Europagestalten der griechischen Mythologie erlangte eine Europa überregionale Bekanntheit und Bedeutsamkeit: die von Phönizien nach Kreta verbrachte Prinzessin. (2) Zwischen ihr und mehreren Heroen und Heroinen wurde mittels Genealogie ein physisches Band von Generationenfolgen gestiftet, das, als biologisches Faktum angesehen, als objektiv gegeben galt; Europa wurde folglich über das Moment der physischen Abstammung fest in eine konstruierte Wirklichkeit eingebunden, welche die Griechen als ,erinnerte' Geschichte begriffen. (3) Fast alle dieser genealogisierend verbundenen Figuren, an deren Anfang die von Zeus begehrte Heroine Io steht, erschienen, wie Europa selbst, als mobile Wanderungsfiguren. Sie bildeten ab dem fünften Jahrhundert eine in griechischer ,Erinnerung، wichtige Großfamilie, die viele Generationen von Mitgliedern umfasste. Deren zwei Hauptmerkmale waren zum einen das, was wir heute „Migration“ nennen: der, so hat es Angelos Chaniotis in Überlegungen zum antiken Kreta formuliert, „dauerhafte permanente oder zumindest langfristige, freiwillige oder erzwungene - Wechsel des Wohnortes einzelner Personen, Familien oder größerer Gruppen“4; zum anderen Eponymenund/ oder Gründungsfunktion.

Europas Einbettung in eine Generationenabfolge, die zahlreiche berühmte mobile Heroinen und Heroen umfasste (u.a. Io, Kadmos, Minos, Rhadamanthys und Sarpedon) dürfte für die Wahrnehmung ihrer Geschichte von großer Bedeutung gewesen sein. War sie doch nicht irgendeine Liebschaft des Zeus, sondern Nachfahrin und Mutter von Heroinen und Heroen, die in Folge ihrer Mobilität im Mittelmeerraum wichtige Funktionen hatten. Diese bedeutende Verwandtschaft trug zur Bekanntheit der Zeusgeliebten maßgeblich bei - zu einer Bekanntheit Europas nicht so sehr als einer Einzelfigur, sondern vielmehr als eines Teils einer sich stetig weiter verzweigenden Mythotopographie, die das ist mit dem Begriff gemeint - einerseits Raum in Mythen und andererseits Mythen in Raum organisierte. Es handelte sich um eine Mythotopographie kultureller und ethnischer Differenz und Diversität, Migration und Interaktion im Mittelmeerraum, bei der Genealogie systembildende Funktion hatte: Die Großfamilie, in der Europa ein wichtiges

4 Chaniotis 2006, 98. 
Glied war, bildete eine Gesamtheit von Elementen, die wechselseitig aufeinander bezogen waren und in Abgrenzung nach außen, ab dem fünften Jahrhundert im Sinne einer hellenischen Identität, eine Einheit bildeten. Zugleich reflektieren die Geschichten der einzelnen Familienmitglieder eine Pluralität von jeweils „kollektiven Identitäten“ im Sinne der Definition, wie sie Jan Assmann in Das kulturelle Gedächtnis gibt: Nicht nur die Geschichte der Europa, sondern auch die Geschichte der übrigen mythischen Figuren, mit denen sie zu einer Familie zusammengebunden wurde, halfen „Bilder, die Gruppen von sich aufbauten und mit denen sich deren Mitglieder identifizierten“ 5 zu etablieren bzw. zu wahren. Von den Geschichten der Wege einer Io, einer Europa, eines Phoinix, eines Kadmos, eines Kilix etc. durch den Mittelmeerraum kursierten zahlreiche Varianten, von denen einige auf lokaler Ebene von identitätsstiftender und -stabilisierender Bedeutung waren (als Beispiel dienen in erster Linie die Ausführungen zu Europa); andere hatten in dieser Funktion über Ortsgebundenheit hinausgehende - ethnische, regionale und, vermehrt ab dem fünften und vierten Jahrhundert v. Chr., panhellenische - Bezüge (als Beispiel dienen unten in 3. die Ausführungen zu Kadmos und Io). Dabei ist auch hier zu beobachten, was in der Identitätsforschung Konsens ist und z. B. von Wolfgang Raible zusammenfassend dargestellt wurde: Identitäten und Alteritäten (,wir-sie') fundieren einander wechselseitig; sie funktionieren über ein Wechselspiel von Inklusion und Exklusion, stehen stets miteinander in Beziehung und sind auf analoge Weise konstruiert. ${ }^{6}$ Viele der Geschichten der mobilen mythischen Heroinen und Heroen der Europafamilie wurden in Abgrenzung nach innen funktionalisiert, etwa im Zuge der Auseinandersetzungen zwischen einzelnen Regionen griechischer Poleis; sie erfuhren aber auch, seitdem sich die Griechen infolge von Kolonisation und kriegerischen Auseinandersetzungen im Gegensatz zu den Barbaren und zu Asien sahen, ab- und ausgrenzende Funktionalisierungen in panhellenischer Optik nach außen und halfen den Griechen, sich als Griechen zu verstehen.

Insofern sind weder die Entführungsgeschichte selbst noch die anderen Geschichten von und um Europa als die begründenden Mythen unseres heutigen Europas zu begreifen. In ihrer gemeingriechischen Vernetzung über Geltendmachung von Blutsbanden aber lassen sich diese Mythen durchaus als eine große komplexe Gründungsnarration der Griechen verstehen - der Griechen als höchst vielfältiger Kulturnation, wie sie sich bereits in archaischer Zeit zu formieren begann. In dem hellenisch besiedelten Mittelmeergebiet markierten die um Europa gruppierten Mythen Eckpunkte griechischer Kultur und wirkten im Einzelnen wie in gemeingriechischem Sinne identitätsstiftend und -stabilisierend. Sie definierten mythotopographisch einen kulturellen und ethnischen Raum, den im Innern wie in den Beziehungen nach außen eine Dialektik von Einheit und Vielfalt kennzeichnet und der stark durch Mobilität geprägt ist. Vieles davon hat die Signatur des heuti-

5 Assmann I997, I30-I60, insbes. I32.

6 Raible I998. 
gen Europa entscheidend mitbestimmt; und nicht zuletzt der Blick zurück - auf den antiken Mittelmeerraum mit seiner Pluralität von Identitäten - hilft den Europäern zu verstehen, wer sie sind.

1.

Die Geschichte der Entführung Europas verläuft in der Form, wie sie nach einem mehrere Jahrhunderte andauernden Kanonisierungsprozess spätestens ab dem vierten Jahrhundert v. Chr. kursiert sein dürfte, verknappt wiedergegeben, folgendermaßen: Schauplatz ist das Ufer der Küste von Tyros oder Sidon im heutigen Libanon. Dort erblickt der griechische Göttervater Zeus (lateinisch Iuppiter) die phönizische Königstochter und entbrennt in sexuellem Begehren. In einen Stier verwandelt, erscheint er Europa und trägt sie, nachdem sie auf seinen Rücken gestiegen ist, über das Mittelmeer nach Kreta. Auf der Insel angekommen, zeugt er mit ihr (nach Homer) zwei oder (nach Hesiod) drei Söhne. Es sind dies Minos, Rhadamanthys und Sarpedon, die - wie u.a. Kadmos, (seit Hellanikos von Lesbos) Europas Bruder, der auszieht, seine Schwester zu suchen - an verschiedenen Orten im Mittelmeerraum zu bedeutenden Gründern werden.7

Der maßgebliche Grund für die Persistenz der Verknüpfung der mit dem Europa liegt in der Gleichheit des Namens der Entführten und des Erdteils, der von Asien und Afrika abgegrenzt wurde. Wie im heutigen deutschen (aber anders als z.B. im englischen) Sprachgebrauch waren die Bezeichnungen im Griechischen und im Lateinischen iden-

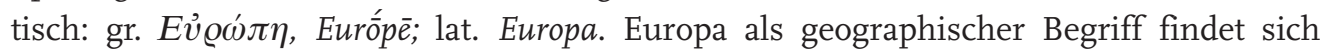
schon vor Herodot: Um 500 v. Chr. war die Welt, wie wir aus den Fragmenten der Grie-

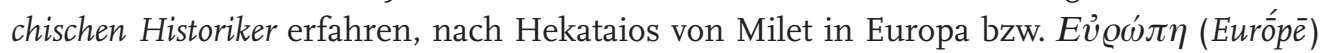
und Asien bzw. 'A oí $\alpha$ (Asía) geteilt (FGrH I,36f.). Herodot führt in seinen Historien bereits einen dritten Erdteil auf: Libyen, das spätere Afrika; als Grenze zwischen Europa und Asien nördlich des Schwarzen Meeres gibt er den Rion oder den Don an (4,42-45). Genannt wird Europa zuerst im Homerischen Apollon-Hymnos (25I, 29I), bezeichnet aber nur Mittelgriechenland, während es bei Herodot häufig im Zusammenhang mit dem Hellespont vorkommt. Zudem bezeichnet es einen Teil Thrakiens. Der Geltungsbereich des Namens war mithin zunächst klein und nicht allgemeingültig festgelegt. Er wurde sukzessive ausgeweitet, im Westen bis zum Atlantischen Meer, im Norden bis nach Skandinavien. Eine politische Idee von Europa im heutigen Sinne gab es im Altertum nicht. Allerdings lassen sich ab dem fünften und vierten Jahrhundert v. Chr. Tendenzen einer ideologisierenden Operationalisierung des Europabegriffs unter anderem in Verbindung

$7 \mathrm{Zu}$ den antiken literarischen Zeugnissen vgl. Bühler I968; zur Genealogie siehe a.a.O. 7-9 und I9-2I. Eine Zusammenstellung antiker Versionen des Europamythos in deutscher Übersetzung findet sich in Renger 2003, I9-59, 222-226. 
mit dem Zusammengehörigkeitsgefühl der Griechen in Abgrenzung von den Barbaren beobachten.

Wohl nicht zuletzt deshalb wurde die Namensgleichheit, wider allen Zweifel des Geschichtsschreibers Herodot, schon in der Antike im Verlaufe der Jahrhunderte regelrecht ausgeschlachtet, wobei eine Vielzahl zunächst existierender Europafiguren mit derjenigen identifiziert wurde, die am bekanntesten werden sollte: die entführte phönizische Königstochter. ${ }^{8}$ Bei vielen prominenten Dichtern, die auf die Verbindung der mythischen Frauengestalt und des Kontinents eingehen, ist von der Tatsache, dass Europa zahlreiche Namenscousinen hatte, nichts zu merken. Europa ist bei ihnen die weithin bekannte Europa: die vom obersten Gott in Stiergestalt nach Kreta verbrachte Prinzessin. Uneindeutigkeit oder Ambivalenzen sind nur insofern zu beobachten, als die Darstellungen der Entführten zwischen einem sich ängstigenden, klagenden Mädchen auf dem Rücken des Übermächtigen und einer den Gott wiederliebenden gefügigen Braut schwanken. Für die Überlieferung besonders wichtig waren neben Moschos, der die Entführung ausführlich in seinem Europa-Epyllion darstellt, insbesondere Horaz und Ovid. Sie flochten in ihre phantasievollen Dichtungen über Europa die Herleitung der Kontinentbezeichnung vom Namen der Entführten ein (Mosch. 2, 8-I5; Hor. carm. 3,27; Ov. fast. 5,603-620). Unter anderem durch die Etymologien des einflussreichen Isidor von Sevilla (Isid. orig. I4,4,I) gelangte die Herleitung dann in die mittelalterlichen Schulbücher, wirkte zumal in Etymologien zur Erklärung der geographischen Bezeichnung „Europa“ fort und findet sich, mit erstaunlicher Häufigkeit, in vielen weiteren Medien bis heute.

Diese Version des Europamythos zur Herleitung des Erdteilnamens, die sich durchgesetzt hat, ist freilich nur eine unter vielen Varianten. Die Sichtung der verschiedenen Testimonien in der antiken Literatur und Kunst ergibt ein weit weniger einheitliches Bild, als es die genannten prominenten Dichter vermitteln. Wie vom Erdteil gab es bei den frühen Griechen auch von der mythischen Frauengestalt Europa unterschiedliche Annahmen, und ein- und derselbe Name bezeichnete Verschiedenes. Die ältesten Quellen berichten keineswegs einhellig von einer einzigen Europa. In der Theogonie nennt Hesiod, der Ende des achten, Anfang des siebenten vorchristlichen Jahrhunderts im boiotischen Askra lebte, Europa in einem Atemzug mit mehreren Schwestern als Tochter des Okeanos und der Thethys (Hes. theog. 357-360). In der Ilias und in Fragmenten, die von Hesiod überliefert sind, wird Europa als Tochter des Phoinix erwähnt (Hom. Il. I4,32I; Hes. fr. I40.I4I,7f. M/W).

Der Blick auf Zeugnisse nach Homer und Hesiod bestätigt den Eindruck, dass die verschiedenen Figuren namens Europa in archaischer Zeit verschiedene Bedeutungen hatten. Sowohl Herodot als auch spätere Dichter und Mythographen sprechen von mehreren Gestalten, die alle Europa genannt wurden, z. B. von der Phoinix- oder von der Agenortochter, die Zeus entführte, aber auch von einer Thrakerin und von einer Okeanide (Hdt. 4,45,4-5; Schol. Eur. Rhes. 29). Europa erscheint in den verschiedenen Testimonien bisweilen als

8 Vgl. dazu auch Dombrowsky I984, 4I-46. 
Heroine, bisweilen als Göttin oder Epiklese einer anderen Göttin, z. B. auch in Gleichsetzung mit Demeter oder Hera, nicht selten in Verbindung mit Heiligtümern, Tempeln, Kulten und Bildern. In der Forschung wurde u.a. deshalb vermutet, Europa sei ursprünglich eine Göttin, eine Mond- oder chthonische Erdgöttin, gewesen 9 - eine Vermutung, die mit etymologischen Erklärungen des Namens, die diesen mit dem Wort „dunkel“ in Verbindung brachten, gestützt wurde. ${ }^{\text {Io }}$ Auch denkbar und m. E. wahrscheinlicher ist, dass die Dimension religiöser Verehrung eine Folge der Prominenz des Mythos war; wissen wir doch, dass die Erzählung von Mythen das Bild der kultisch verehrten Gottheiten nicht unerheblich formte und beeinflusste. Wie auch immer es sich verhalten haben mag: In welchem Verhältnis zueinander Erzähl- und Kultpraxis in Europas Fall im Einzelnen standen, erlaubt die Quellenlage nicht zu entscheiden. Gewiss ist bei Betrachtung der überlieferten Zeugnisse nur, dass Erzähl- und Verehrungskultur stark ineinander diffundierten. Viele der, nach Darstellung der Dichter, lokalspezifisch signifikanten Europagestalten erfuhren, insbesondere im zentralgriechischen Boiotien und auf Kreta, ortsgebundene kultische Verehrung. ${ }^{\text {II }}$

Eine Auswahl aus den vielen Zeugnissen Europas mag die Verschiedenheit ortsgebundener religiöser Signifikanz verdeutlichen:

Der in Boiotien geborene Pindar etwa erwähnt eine Quelle namens Europa im Zusammenhang mit den Flüssen Acheloos und Melas (Pind. Paean fr. 70,249b), und am boiotischen Kephisos soll Europa, die Tochter des Tityos, einen Sohn geboren haben (Pind. P. 4,44-47). Ferner findet bei Pindar, wie später auch bei Apollonius Rhodius und bei Pausanias im Zusammenhang mit der boiotischen Lokalsage von Europa, eine Höhle Erwähnung, in der Zeus Europa versteckt habe (Pind. P. 4,46; Apoll. Rhod. I,I79-I84; Paus. 9,I9,I). Von Pausanias haben wir zudem eine Notiz über einen Tempel der Demeter mit Beinamen Europa und des Zeus Hyetios, der im Hain des boiotischen Heros Trophonios von Lebadeia gestanden haben soll (9,39,4-5). Der Priester des Trophonios soll dieser Göttin als der Amme des Trophonios, der als Orakelgott verehrt wurde, geopfert haben. Sowohl die Höhle als auch die Verbindung mit der Fruchtbarkeitsgöttin Demeter, noch dazu in Nachbarschaft des Orakelgottes Trophonios, dessen Befragung in einer Höhle stattfand, legen einen chthonischen Charakter der Figur nahe. Es wird daher, wie oben bereits angedeutet, auch von der „böotischen Erdgöttin Europa“ gesprochen. Auf Kreta hingegen erfuhr die Europa Hellotis Verehrung. Ihr zu Ehren wurden, wie aus Theophrasts Historia plantarum (I,9,5), Plinius' Naturalis historia (I2,II) und Athenaios (I5,22,678b) zu schließen ist, alljährlich die Hellotia gefeiert; in ihrem Verlauf wurde ein Myrtenkranz mit den Gebeinen der Europa herumgetragen (De Seleuco Homerico. Fr. 52 Müller ${ }^{\mathrm{I} 2}$ ). Kretische

9 Vgl. z.B. Escher-Bürkli i907.

Io Vgl. Helbig I884-I886, I4I7f.; Escher-Bürkli I907, I287; Ninck I945, I9; Pfligersdorffer I966, 965; Schumacher I990, I2; Musäus 2009, $344 \mathrm{f}$.

II Vgl. dazu (mit Nachweisen) Zahn I983, 70-74; Schumacher I990, I6.

I2 Mueller I891, 47. 
Münzen insbesondere aus Gortyn zeigen ab dem fünften Jahrhundert v. Chr. Europas Bild, oft in Gestalt einer Baumnymphe, woraus auf ähnliche Traditionsstränge wie im Falle der Okeanide bzw. boiotischen Nymphe zu schließen ist. Von wieder einer anderen Europa, nämlich einer Thrakerin, nach welcher der Kontinent benannt sei, berichtet Hegesippos von Mekyberna. Er nimmt die seinerzeit gängige Verknüpfung des Europa- mit dem Kadmosmythos auf. In Fragment 6 im Scholion zu Euripides, Rhesus 28 heißt es, von der Namensgleichheit irregeführt, sei Kadmos den falschen Weg gegangen, und nach dieser Europa sei alles Land im Norden benannt. Dass Europa in Thrakien auch in der Kaiserzeit eine wichtige Rolle spielte, bezeugen ebenfalls u.a. Münzprägungen. ${ }^{13}$

Am frühesten kursierte im gemeingriechischen Erzählschatz die von Homer und Hesiod genannte Tochter des Phoinix. Die Bezeichnung Europas als Phoinix-Spross lässt zwar nicht zwingend auf phönizische Herkunft schließen (Phoinix ist kein phönizisches Wort, es findet sich als geographischer Name in Griechenland von Epirus über die Peloponnes bis zu den Inseln und Lykien; als Appellativum bedeutet es, neben „Phönizier“ und einem sehr speziellen Vogel, „Purpur“ und „Dattelpalme“); ;4 Phönizien gilt aber in den meisten Quellen als Entführungsort Europas, Phoinix als König von Sidon oder Tyros und Europa daher als Phönizierin. An beiden Orten wurde an Europa gern angeknüpft: In Sidon gab es z. B. in späterer Zeit einen Tempel der Europa-Astarte, woher ab dem zweiten Jahrhundert v. Chr. Münzen mit dem Europa-Motiv bekannt sind; und in der pseudolukianischen Schrift De dea Syria heißt es, laut einem einheimischen Priester aus Sidon sei der Tempel der Astarte dort nach Europas Verschwinden errichtet worden (De dea Syria 4). In Tyros z.B. diente als konkrete Form der memoria, der Erinnerung an Europas Entführung, ein Fest. ${ }^{15}$

Spätestens ab dem fünften Jahrhundert v. Chr., als in der Mythologie maßgebliche Familienzusammenführungen stattfanden und die Griechen ihre Frühgeschichte, als die sie die mythische Zeit verstanden, chronologisch ordneten und festlegten, scheinen die diversen auf Boiotien, Thrakien, Kreta und Phönizien bezogenen Aussagen übereinander geblendet und die diversen Europagestalten mit der vom Göttervater Zeus in Stiergestalt entführten Phönizierin gleichgesetzt worden zu sein. Lokalsagen und -kulte dürften weiter bestanden haben. Im allgemeinen Erzählschatz der Griechen setzte sich aber die oben umrissene Fassung der Entführung durch. Verschiedene mythische und religiöse Vorstellungen und Gebräuche griechischen und orientalischen Ursprungs, lokaler, regionaler und panhellenischer Bedeutsamkeit wurden während dieses Kanonisierungsprozesses amalgamiert. Schließlich avancierte Europa zur Eponyme einer geographischen Einheit, die als nord- bzw. zentralgriechische Region ihren Anfang nahm und dann sukzessive ausgeweitet, wenn auch in der Antike nie genau geographisch umrissen wurde.

I3 Vgl. dazu Zahn I983, $78 \mathrm{f}$.

I4 Beekes 2004, I8I-I83 (ausführliche Erörterung der Wortherkunft und -semantik).

I5 Vgl. dazu Bühler I968, 45f.; Zahn I983, 76 f. 
Parallel zu diesem Kanonisierungsprozess und der Ausweitung des geographischen Begriffs Europa ab dem fünften Jahrhundert v. Chr. (beide Entwicklungen können in diesem Beitrag nur knapp und nicht so ausführlich, wie es gebührte, dargestellt werden) kam es, wie allgemein in der griechischen Mythologie, zu einem zunehmenden Ausbau der Generationenabfolge der Europafamilie. Mit dem fünften Jahrhundert v. Chr. entstand in Griechenland eine neue Prosagattung, deren Gegenstand die altüberlieferten Erzählungen von Göttern und Heroen waren. Eine Vielzahl von Autoren, von denen nur der Name oder wenige Fragmente auf uns gekommen sind - z.B. Akusilaos von Argos, Pherekydes von Athen und Hellanikos von Lesbos, ferner Damastes von Sigeion, Hippias von Elis, Polos von Akragas - sammelten und verglichen, prüften und ordneten die tradierten Erzählungen und Genealogien, um sie zu (re)konstruieren und zu komplettieren. Wie andere Gestalten der griechischen Mythologie wurde im Zuge dieser ordnenden Aktivität auch Europa in einem erzählerisch und geographisch weiträumigen Geflecht auf immer vielfältigere Weise vernetzt. Sie wurde Gegenstand einer genealogischen Mythotopographie, die u.a. durch Schaffung von mythischen Memorialorten (Orten der Erinnerung an mythische Heroen und Heroinen) Raum und Mythen integrativ-organisierend ordnete. Dabei griff das, was Max Weber in seinen Ausführungen zu ethnischen Gemeinschaftsbeziehungen in Wirtschaft und Gesellschaft, ,subjektiven Glauben an eine Abstammungsgemeinschaft“ nennt. Wirksam wurde eine Art „Stammverwandtschaftsglauben“, wie er, so Weber, u.a. durch „Erinnerungen an Kolonisation und Wanderung“ gestützt wird. ${ }^{16}$ Anders formuliert: In bestimmten Gruppen wurde abgeleitete Herkunft in Form von Verwandten in auf- und in absteigender Linie geglaubt. Sie wurde als biologisches Faktum, also Gegebenheit der Natur erinnert und mittels dieser, mit Peter L. Berger und Thomas Luckmann gesprochen, „Verdinglichung“ zu einer wirksamen Potenz für „gesellschaftliche Konstruktion der Wirklichkeit“ zu Identitätszwecken. ${ }^{\text {77 }}$ Was im Geiste kreiert worden war, galt als real.

Allgemeine genealogische Systematisierung unter Vernetzung verschiedener Mythenstränge war im antiken Griechenland überaus verbreitet. Sie begegnet nicht nur in den homerischen Katalogen, sondern auch, in besonders verdichteter Form, in den Ehoiai, die den Heroenmythos in systematisch katalogisierter Gestaltung der Stammbäume darstellen, und fand auch in Hesiods Theogonie elementar Anwendung. Ein Grund hierfür könnte darin gelegen haben, dass katalogische Darstellung Ausweis der Realienkompetenz eines Dichters war. In jedem Fall hatte die genealogische Systematisierung eine reale Basis. Genealogie als Ableitung der Herkunft in Form von Ahnenreihen in aufsteigender und Nach-

I6 Weber 1972, 237 (Kap. IV, 『2: „Entstehung ethnischen Gemeinsamkeitsglaubens. Sprach- und Kultgemeinschaft").

I7 Berger/Luckmann I966. 
kommen in absteigender Linie war in frühen, durch Familienverbände geprägten Gesellschaften ein häufig verwendetes Mittel der Legitimation und (vermeintlich) historischen Erinnerung. Genealogisch miteinander verknüpfte Personen gehörten zu einer „Verwandt-

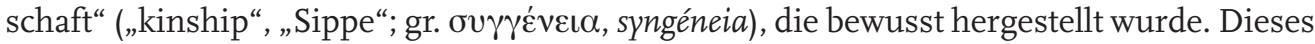
Verfahren der Verwandtschaftsherstellung wurde in Griechenland seit früher Zeit intensiv gepflegt. D.h., der geflechtartigen Verbindung einzelner Götter und Helden sowie ganzer Gruppen, wie sie Hesiod vornahm, liegt nicht etwa ein rein künstliches Prinzip zugrunde, das keinen historischen Bezug hat. Was auf die Welt der Götter und Helden projiziert wurde, war nicht erdacht, sondern fand bis tief in klassische Zeit hinein real gesellschaftliche Anwendung: Eine gängige Praxis vor allem griechischer Aristokraten, die übrigens auch Homers Epen abbilden, war es, sich mittels Verschwägerung und Gastfreundschaft mit Personen auch aus anderen Regionen und Ethnien zu verbinden. ${ }^{18}$ Durch Darstellung dieser Vorgänge auf Bildmedien und Integration in die griechische Literatur wurden sie zum Gegenstand allgemeingriechischer Erinnerung.

Es überrascht nicht, dass Genealogie in klassischer Zeit, als Ethnizität bei den Griechen zunehmend wichtig und politisch funktionalisiert wurde, regelrecht, in Mode kam ' ${ }^{19}$ Unter der Impression der Perserkriege wurde der gedachte Gegensatz von "Gräzität“ und „Barbarität“, wie von Hans-Joachim Gehrke mehrfach dargelegt, ${ }^{20}$ zur „Wir-Sie“-Standardantithese. Viele bedeutende mythische Figuren, die in der archaischen Zeit noch als Griechen gegolten hatten, wurden nun wieder, rebarbarisiert' und als Immigranten gehandelt Edith Hall hat das z.B. fürs attische Drama nachgewiesen ${ }^{2 I}-$, um später, bei den hellenistischen Mythographen, unter denen die Arbeit mit Genealogie einen Gipfelpunkt erreichte, wieder ,hellenisiert' zu werden. Je nach Genre und soziokulturellem Kontext zielten die Darstellungen auf unterschiedliche Lokalitäten und Gruppen, und waren von den lebensweltlichen Bedingungen, unter denen sie verfasst wurden, geprägt. Autoren des fünften und vierten vorchristlichen Jahrhunderts etwa standen maßgeblich unter dem Eindruck und Nachwirken der Ereignisse der sogenannten Großen Kolonisation und der großen Kriege, erst der Perserkriege, dann der Peloponnesischen Kriege. Seit dem Hellenismus hingegen fanden in den Darstellungen die Expansions- und Migrationsschübe sowie Städtegründungen, die aus dem rund zehnjährigen Eroberungsfeldzug Alexanders des Großen resultierten, ihren Reflex. Es kam nun zu immer mehr mediterraner Mobilität auch ohne kriegerischen Anlass; zur Kommunikation der Poleis untereinander, wobei indigene Gemeinschaften freiwillig den Status griechischer Poleis annahmen. Diese Entwicklung fand nachhaltigen narrativen Niederschlag: Durch Erzählen zahlreicher Geschichten wurden die ursprünglich nichtgriechischen Gemeinschaften in die griechische

I8 Vgl. dazu z. B. Herman I987, insbes. 3-8, I42-I46; Coldstream I993.

I9 Vgl. dazu z.B. Gehrke 2005; Miller 2005.

20 Z.B. Gehrke 2003.

2I Hall ig89. 
Geschichte integrativ-hellenisierend eingebunden - nicht zuletzt mittels Konstruktion von Verwandtschaftsbeziehungen.

Eine besonders wertvolle Quelle, die das Prinzip großflächigen Verknüpfens verwandtschaftlicher Beziehungsgeflechte zur Darstellung bringt, ist die sogenannte Bibliotheke oder Bibliothek Apollodors, ein umfangreiches mythographisches Handbuch. Es zeigt deutlich, wie das genealogische Prinzip des Ordnens und Vervollständigens wirkt. Europas Familie wird im dritten Buch unter den Nachfahren Ios bzw. ihres Vaters Inachos geführt. Ihre Blutsbande reichen u.a. bis zum thebanischen Herrscherhaus (3,I-95); mithin sind auch Ödipus und seine Kinder mit ihr auf gemeinsame Abkunft festgelegt. Die Bibliotheke wird Apollodor aus Athen, dem Gelehrten, der im zweiten Jahrhundert v. Chr. lebte, zugewiesen, stammt aber aus der Kaiserzeit, möglicherweise aus dem ersten oder zweiten Jahrhundert n. Chr. Umstritten ist, ob das Werk indirekt auch auf Apollodor zurückgehendes Material enthält. In jedem Fall bietet es, bei aller gewiss auch individuellen Gestaltung der Mythologie, einen Blick auf archaische Schichten des Dargestellten. Zitiert es doch eine beträchtliche Menge älterer Schriftsteller wie etwa die oben erwähnten Autoren Akusilaos von Argos und Pherekydes von Athen, deren Darstellung zum Teil in vorhomerischer, mündlicher Tradition wurzelt.

In Hinblick auf die Frage nach Mobilität in der Antike ist es signifikant, dass die Bibliotheke viele Verwandte der Europa, die im Laufe der Jahrhunderte in den immer weiter ausgearbeiteten genealogischen Stemmata ihrer Familie einen Platz fanden, gesammelt so zeigt, wie sie auch in vorausgehenden Einzeldarstellungen erscheinen: als bedeutende Migrationsfiguren mit Eponymenfunktion. Fast alle Figuren in Europas Familie wandern, und kaum eine wird nicht entweder Stammvater bzw. -mutter oder ist eponymer Gründer

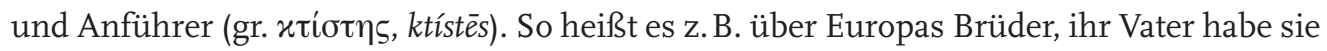
nach dem Verschwinden ihrer Schwester auf die Suche geschickt, mit der Auflage, erst zurückzukehren, wenn sie sie gefunden hätten $(3,3)$. Die Brüder hätten, als die Suche vergeblich gewesen sei, den Plan ihrer Heimkehr verworfen und sich, der eine hier, der andere dort, angesiedelt: „Phoinix in Phoinikien, Kilix nahe bei Phoinikien; er nannte das ganze Land, das beim Fluss Pyramos liegt, nach sich selbst Kilikien; Kadmos und seine Mutter Telephassa ließen sich in Thrakien nieder. Ebenso siedelte sich auch Thasos an, der in Thrakien eine Stadt namens Thasos gründete“ $(3,4)$. Europa habe, so fährt Pseudo-Apollodor fort, Asterios, den Herrscher von Kreta geheiratet und ihre drei Söhne Minos, Sarpedon und Rhadamanthys aufgezogen. Die beiden ersteren aber seien in Streit um den Knaben Miletos geraten, den sie beide liebten; und der Knabe sei nach Karien gegangen, wo er die Stadt Miletos begründet habe. Sarpedon habe für Kilix, der mit den Lykiern Krieg führte, gekämpft, wobei ihm ein Teil des Landes zufiel und er König von Lykien wurde. Rhadamanthys sei nach Boiotien gegangen, wo er Alkmene geheiratet habe. Nur Minos sei auf Kreta geblieben und habe dort zahlreiche Nachfahren gezeugt (3,5-7). Pseudo-Apollodors katalogische Darstellung zeigt beispielhaft sehr schön, wie mittels Genealogie Vergangenheit als ein in sich bewegtes und kausal verknüpftes Zeit-Raum-Gebilde konstruiert wird, 
in dem jede Person bzw. Figur eine Position einnimmt und eine Identität hat bzw. eine solche stiftet. Beim Lesen entsteht unweigerlich der Eindruck, dass viele der Figuren - vor allem die Eponymen, deren Name als Inbegriff und Zentralsymbol der Großgruppen, die ihn kollektiv trugen, jeweils von dem des Kollektivs hergeleitet war - im Wesentlichen Kunstfiguren waren.

Die Eponymenfunktion der Migrationsheroen und -heroinen aus Europas Familie ist in Texten und Bildern außerhalb der Bibliotheke schon in archaischen und klassischen Quellen (aber auch hellenistischen und solchen der Kaiserzeit) ebenfalls reich dokumentiert. Überblicksartikel zu den einzelnen Figuren in einschlägigen Sachlexika wie z.B. Paulys Realenzyklopädie der klassischen Altertumswissenschaft zeigen dies sehr deutlich. Aus ihnen geht auch hervor, dass an Europas Verwandte seit allerspätestens dem vierten Jahrhundert v. Chr. regelmäßig Gründungsmythen geknüpft wurden: Aus den verschiedenen Wanderungen der Familienmitglieder resultierten Niederlassungen, Errichtungen und Besiedelungen verschiedener Stätten - Kultplätze, Tempel, Städte u.a.; sie umgriffen das Mittelmeer- und Schwarzmeergebiet, also genau den Raum, in dem Griechen sich im Verlauf der wechselvollen Migrations- und Expansionsprozesse der sogenannten Großen Kolonisation von Mitte des achten Jahrhunderts bis etwa 550/500 v. Chr. in Städten nach griechischem Muster ansiedelten. Genannt seien hier, als zwei Beispiele bei prominenten Autoren, Io und Europa selbst: Europa gelangte laut Herodot 4,45,5 von Phönizien nicht nur nach Kreta, sondern von dort auch nach Lykien. Und ihre Ahnin Io wandert in Aischylos' Prometheus Desmothes von Argos über Dodona erst zum Ionischen und dann zum Schwarzen Meer, begegnet Skythen und Amazonen und kommt schließlich nach Ägypten; die Nachfahren, die sie auf der Reise hinterlässt, kehren zum Teil nach Argos zurück. An Io wird mit besonderer Transparenz deutlich (s. hierzu auch weiter unten): Je größer die Mobilität einer Figur mythologischer Darstellung nach war, je mehr Orte sie passierte, desto vielfältiger waren sowohl die an sie geknüpften Mythen, Kulte und Stätten auf jeweils spezifisch lokaler Ebene als auch darüber hinausgehende Bezüge von ethnischer, regionaler und panhellenischer Dimension. Eine Figur, die viel wanderte, war besonders anschlussfähig. Verschiedene Gruppen bezogen sich auf sie, je nachdem wo und wie sie Ansatzmöglichkeiten sahen und welcher Art ihre Aussagebedürfnisse waren: Die an eine Migrationsfigur geknüpften Mythen und Kulte konnten funktionalisiert werden, um freundschaftliche Bande und friedliche Koexistenzen verschiedener Gruppen zu fördern und bei konfligierenden Gebietsansprüchen zu vermitteln oder, umgekehrt, Feindschaft und Besitznahmen zu rechtfertigen.

\section{3.}

Wie bereits deutlich geworden sein dürfte, ist die Koinzidenz der Mittelmeer- und Schwarzmeer-Topographie in Geschichte und Mythos kein Zufall. Hinter der Vorstellung der primordialen Gestalt des eponymen Gründers standen reale Ereignisse und komplexe 
Prozesse, die von Interessen wie Handel, Besitz, Herrschaft, Landnahme und Besiedelung und deren jeweiliger Legitimierung bestimmt waren. ${ }^{22}$ Griechen verließen im Zuge der Großen Kolonisation die Peloponnes und gingen nach Nordafrika, Kleinasien, in die nordpontischen Gebiete und den Vorderen Orient, nach Südfrankreich und Spanien, Dalmatien, Sizilien und Süditalien. Die Grenzen, welche die Eponymen und Gründer in der Mythologie passierten und neu setzten, die Gebiete, welche sie durchmaßen, waren mithin tatsächlich viel durchreist und durchwandert. Die wechselvollen Zeiterfahrungen prägten die Erzählkultur derart, dass der geographische Raum zugleich ein Raum mythotopographischer Identitätsbildung im Spannungsfeld von Einheit und Pluralität war. Die Griechen spannen in ihm und spannten durch ihn - während sie jeweils an verschiedenen Gruppenidentitäten arbeiteten, aber auch sukzessive im Sinne einer gemeingriechischen Sache ihre Identität als „Hellenes“ mit einer gemeinsamen Sprache und einem gemeinsamen verbindlichen Pantheon herausbildeten - narrativ ein Netz von Bewegungen und Beziehungen. Dabei wurden verschiedenste Komponenten gegenwärtiger Ordnungen als frühe oder gar früheste Setzungen in die Vergangenheit oder gar in einen imaginären Ursprung verlegt; und genealogische Beziehungen bildeten den Nexus der historisierenden Entwürfe. Für die Phänomene Mobilität und Migration bedeutete das in der erzählerischen Darstellung: Gewandert wurde bereits früher - von den Vorfahren, die schon vor langer Zeit maßgeblich gründend und zeugend tätig waren.

Die Forschung hat diese narrative Rückprojektion in die Vergangenheit kontrovers diskutiert. Jonathan M. Hall z. B. geht in seiner Diskussion von Wesen und Ausdrucksformen ethnischer Identität im antiken Griechenland so weit, die Historizität bestimmter literarisch überlieferter geographischer Bewegungen, vor allem der Ionischen Wanderung, grundsätzlich zu bezweifeln. ${ }^{23}$ Die hier angestellten Überlegungen wollen sich dieser Extremposition nicht anschließen. Betont sei: Wir wissen nicht, wie die diversen Wanderungsbewegungen von der Bronzezeit über die sogenannten Dark Ages bis in die Archaik im Einzelnen verliefen; Konstruktionsversuche werden wohl stets eine hypothetische Note behalten. Wir können aber davon ausgehen, dass Erzählungen von Wanderungen tatsächlich historische Bewegungen widerspiegeln. Damit soll nicht etwa behauptet werden, dass bestimmte Mythen umfassende Wanderungen von Großstämmen wie Aiolern, Ioniern und Dorern abbilden; dass solche Wanderungen Konstrukte sind, ist inzwischen Konsens. ${ }^{24}$ Vertreten sei vielmehr die These, dass in Erzählungen von Wanderungen reale und erfundene Ereignisse in jeweils verschiedenen Mischverhältnissen verbunden sind; dass es Migrationen gegeben hat, ihr historischer Kern aber nicht mehr eruierbar ist. Dieser Befund ist insofern unproblematisch, als - das hebt, unter Anschluss an Jan Assmanns Theorie des „Kulturellen Gedächtnisses“, auch Hans-Joachim Gehrke hervor - der Erinne-

22 Prinz I979, insbes. I-I5 (Einleitung).

23 Hall i997.

24 Vgl. dazu z.B. Gehrke 200o; Hall 2002, 56-89. 
rungsfaktor wesentlich ist; und der ist letztlich historizitätsunabhängig. Bestimmte Erinnerungen sind für Gemeinschaften bzw. soziale Gruppen, die als solche nur existieren, wenn sie die Lebensspannen der ihnen zugehörigen Individuen überschreiten, konstitutiv; und sie werden beständig durch Rituale, Denkmäler, Bilder und nicht zuletzt Erzählungen, gepflegt. ${ }^{25}$ Dabei zählt nicht reale, sondern nur erinnerte Geschichte bzw. wird, mit Assmann gesprochen, „faktische Geschichte in erinnerte und damit in Mythos transformiert". ${ }^{26}$

Die Migrationsmythen von Europa und anderen Eponymen ihrer Familie gehörten zu eben dieser Art von Erzähl- und Erinnerungskultur. Es sind Erzählungen, mit denen qua Erinnerung das betrieben wurde, was Gehrke mit Blick auf die Griechen „Vergangenheitsrepräsentation“ nennt: narrative Erzeugung von großer temporaler Tiefe durch Amalgamierung von Mythos und Geschichte zum Zwecke von Ortsbestimmung, Selbstvergewisserung sowie Identitätsstiftung und -wahrung. ${ }^{27}$ Die Funktionsspanne der einzelnen mobilen Familienmitglieder Europas war dabei sehr breit. Sie wurden innerhalb der Vielzahl verschiedener Gruppen und Großgruppen in Kleinasien und dem Vorderen Orient, auf Kreta und auf dem griechischen Festland durch die Jahrhunderte hindurch auf mannigfaltige Weise in Anspruch genommen. Wie Europa - zu ihr wurden oben in Bezug auf einen ausgewählten Bereich ihres Vorkommens, nämlich dem lokaler religiöser Verehrung, einige Beispiele gegeben - fungierten auch ihre Verwandten als lokale, regionale und kommunale, innerstädtische und über einzelne Poleis hinausreichende Referenz- und Identitätsfiguren, die für eine Diversität von Erinnerungen an frühe Zeiten verschiedentlich vereinnahmt wurden.

Ein Beispiel für diese vielfältige Funktionalisierung ist Kadmos. Die Erzählung davon, wie er auf der Suche nach Europa aus Tyros (Hdt. 2,49,3; Eur. Phoen. 639) bzw. Sidon (Eur. Phrixos TrGF 8I9 ${ }^{2}$ ) nach Griechenland gelangt, wo ihm das Orakel in Delphi rät, die Suche aufzugeben und einer Kuh zu folgen, die ihn schließlich nach Boiotien führt, wo er Theben gründet, hat viele verschiedene Phasen der Entwicklung und Vereinnahmung durchlaufen. ${ }^{28}$ Kadmos' Geschichte ist ein bezeichnender Reflex der Tatsache, dass jeder Grieche zwar zu einer spezifischen Polis gehörte, diese Polis aber durchaus Verwandtschaftsbeziehungen mit anderen Poleis und daher Zugang zu deren Sprache, Sitten, Ritualen und Mentalitäten haben konnte. Der Kadmosmythos bewährte sich in der Überlieferung zunächst als Zeugnis von Thebens Position als Hegemonialmacht in Boiotien und erhielt in dieser Bedeutung durch genealogische Vernetzung, zumal vor dem Hintergrund des griechisch-persischen Gegensatzes, gesamtgriechische Signifikanz. Bei näherer Betrachtung der Entwicklung des Mythos fällt auf, dass die frühen Quellen, Homer und He-

25 Gehrke 2008.

26 Assmann I988; Assmann I997, I9-21, 24, 48-66.

27 Gehrke 2008, Io.

28 Vgl. dazu z. B. Miller 2005, 79-84; Kühr 2006, passim. 
siod, von Kadmos' Verwandtschaft mit Europa noch nichts wissen. Die Annahme phönizischer Herkunft des Kadmos wurde erst im fünften Jahrhundert v. Chr. vorherrschend. In den Testimonien bis zum sechsten vorchristlichen Jahrhundert erscheint Kadmos als Stammvater des Herrschergeschlechts auf der thebanischen Kadmeia, Thebens Akropolis. Dass er als Phönizier galt, ist erst durch Bakchylides (Dithyr. 3,I8; 5,46) belegt; und erst Hellanikos weist, wie oben erwähnt, Kadmos als Bruder der Europa aus (Hellanikos FGrH 4 fr. 5I), deren eigenes Stemma als Phoinixspross nach Kleinasien deutet. ${ }^{29}$ Europa und Kadmos wurden offenbar über Zuweisung zu einem gemeinsamen Vater, Agenor, dem König in Sidon, genealogisch verbunden, mit der Folge, dass Kadmos als mobiler Phönizier, seine Gründung Thebens als koloniale Ktisis und die phönizische Stadt Sidon als Thebens Metropolis erschienen. Darüber hinaus ließ sich Kadmos als Identifikationsfigur von den Argivern in Dienst nehmen - angesichts der Kämpfe Thebens mit Argos, wie sie sich im Sagenstoff der Sieben gegen Theben und ihrer Söhne, der Epigonen, andeuten, eine pikante Variante der Mythenvereinnahmung. Agenor stammte von der argivischen Io ab, der Tochter des Königs Inachos von Argos (Aischyl. Prom. 589 f.; Bakchyl. I9,I8 u.a.). So betrachtet, erscheint Kadmos als Argiver ${ }^{3 \circ}$ und Theben als "[home] away from home“, wie Carla Antonaccio den Begriff Apoikia in ihren Überlegungen zur Bildung hybrider Identitäten während der Großen Kolonisation treffend übersetzt: als Siedlung bzw. Neugründung der Argiver außerhalb des Gebietes ihrer Mutterstadt. . $^{\mathrm{I}}$

Wie Kadmos lokalisierten die Griechen auch Io in ihrem (durch weitläufige Verwandtschaft strukturierten) mythischen Vergangenheitsraum, den sie als ihre Geschichte ansahen. Wie Kadmos - auf seine Abstammung von Io weist u.a. Bakchylides hin (Bakchyl. 19,15-43, insbes. 39-43) - ist Io eine wichtige Figur in der Mythhistorie der Griechen. Während der als Einführer der Schrift und Kulturbringer gefeierte Burgherr durch seinen Aufbruch aus Phönizien, Wanderung über Delphi und schließlich die Gründung des thebanischen Königshauses in griechischer Wahrnehmung für Ursprünge ,griechischer Kultur' steht, 32 ist es bei seiner Ahnin ihre Funktion als frühe Repräsentantin der Argiver, die sie zum figural-topographischer Marker ,griechischer Zivilisation' macht: Sie weist hin auf die Argolis mit ihren mykenischen Burganlagen, deren Ruinen den Griechen archaischer und klassischer Zeit zur Erinnerung an eine heroische Vergangenheit dienten. Dabei ist, wie Kadmos auf seine Weise, auch Io ein Beispiel für Verwandtschaftsbeziehungen von Griechen mit Griechen anderer Regionen und Poleis - und zugleich ein Beispiel der Bedeutung von Genealogie für die Bestimmung der eigenen Herkunft und Identität. Die verbindende Funktion qua Rückerinnerung, die ihr als ,Migrantin“ eignete, wurde seit frühester Zeit vielfach geltend gemacht. Ios Bekanntheit belegen bereits Epen des sieben-

29 Vgl. dazu Kühr 2006, 9I-93, 100-106.

30 In diesem Sinne Scheer I993, 3I9 (zu Kadmos 307-320).

$3 \mathrm{I}$ Antonaccio 2007, 204 (zu den Termini Metropolis und Apoikia).

32 Vgl. dazu Kühr 2006, I03-106. 
ten Jahrhunderts v. Chr., Aigimios (Hes. fr. 294; 296), Danais (EpGF p. I4I) und Phoronis (EpGF p. I53-I55); auf ihnen beruhen sowohl die Berichte der Historiker Akusilaos von Argos (FGrH 2 fr. 26f.), Pherekydes von Athen (FGrH 3 fr. 67) und Herodot (Hdt. I,I; 2,4I; 3,27) als auch lyrische und dramatische Darstellungen (Pind. N. 4,35; Bakchyl. I9; Aischyl. Suppl. 29I-3I5; Prom. 56I-900; Soph. Inachos TrGF IV fr. 269a; Eur. Phoen. 247f.; 676-68I).33 Die Testimonien weisen auffällige Strukturäquivalenzen zu Europas Geschichte auf: Wie Europa musste die argivische Herapriesterin ihre Heimat verlassen, weil der Göttervater sie begehrte; wie Europa gründete Io mit Zeus ein neues Geschlecht.34 Es sei nicht versäumt zu erwähnen, in welchem bedeutungsvollen Zusammenhang sich Io und Europa, jene nach Ägypten, diese nach Kreta gekommen, zu Beginn der Historien Herodots befinden: Die beiden werden hier zur tieferen Ursache für die politischen Spannungen gemacht, die 490 und $480 / 79$ in den gegen Athen und Hellas gerichteten Perserfeldzügen kulminierten.35 Der Aussage persischer Gewährsleute folgend, will Herodot die Argiverin von Phöniziern nach Ägypten entführt wissen und macht sie damit zum ersten Glied einer Kette von Frauenrauben in mythischer Zeit, in der Europas Entführung aus Phönizien als Gegenschlag der Griechen und der Trojanische Krieg als Endresultat erscheinen (Hdt. I,2,I). Auf diese Weise definiert er den Konfliktbereich, in dem Perser und Griechen gegeneinander antraten und sich von einander abgrenzten.

Zur Prominenz der Argiverin trug ihr besonders hoher Mobilitätsgrad bei: Io war gleichsam Prototyp der mythischen Migrationsfigur; keiner ihre Nachfahren reichte an sie heran. In eine Kuh verwandelt, durchwanderte sie Nordgriechenland, das ionische Meer, das nach ihr benannt sein soll (Aischyl. Prom. 839-84I), ebenso wie den Bosporos (Aischyl. Prom. 733) und gelangte über Skythien und Asien nach Ägypten (Aischyl. Suppl. 540-573; Prom. 707-735; 790-8I5; 829-847). Wohin die autochthone Argiverin auch kam, hinterließ sie Nachkommen, die in der Zusammenschau als ein Volk erscheinen. In Ägypten schließlich gebar Io dem Zeus den Epaphos, der bei Aischylos beispielhaft als wichtiges Bindeglied zwischen seinen Eltern (als Urahnengeneration) und den Folgegenerationen zwischen Vergangenheit und Gegenwart - erscheint: In ununterbrochener Kette stammen von ihm erst Libye (oder Libya), dann Belos und schließlich Aigyptos und Danaos ab (Aischyl. Suppl. 3I3ff.; Prom. 848-858 u.a.). Dessen Töchter, die Danaiden, verweisen in den Hiketiden bei ihrem Gesuch um Asyl in Argos nachdrücklich auf ihr weit in die Vergangenheit reichendes Geschlecht, ihre argivische Abstammung (von Io und deren Sohn Epaphos), und somit auf die Verbindung „Argos-Ägypten“. Aischylos thematisiert auf diese Weise u.a. die Problematik der Generationendependenz im Verhältnis von Genos und Polis. ${ }^{36}$ Freilich war der alte attische Tragiker nicht der einzige, der, über den Mythos von Io

$33 \mathrm{Zu}$ weiteren Quellen s. z. B. Eitrem I9I6 und Yalouris I990.

34 Auf die Analogien der beiden Mythen hat die Forschung mehrfach hingewiesen, vgl. zuletzt Kühr 2006, 94 f.; Kühr 2009, IIIf.

35 Vgl. dazu z.B. Cobet I996, 408.

36 Ausführlich Föllinger 2003, 218-235. 
und Epaphos, die alte Verbindung „Argos-Ägypten“ aufgriff. Die Verbindung wurde zum Zwecke eigener Aussagen immer wieder thematisiert (vgl. z. B. auch Pind. N. Io,5). Zurückzuführen ist dies - u.a. - auf das Interesse der in Ägypten siedelnden Griechen, insbesondere Kyrenes, die mit dem Mythos Anteil am griechischen Kosmos zu reklamieren suchten.37

Wie den Vorfahren Europas kommt auch ihren Nachkommen, den Söhnen, die aus der Verbindung mit Zeus hervorgingen, im mythischen Vergangenheitsraum der Griechen eine wichtige Position zu. Deuten Europas Ahnin Io und Libye, die autochthone Frau bzw. Königstochter aus Ägypten, zurück auf frühe kulturelle Verflechtungen im Mittelmeerraum, in denen Ägypten eine maßgebliche Rolle spielte, so sind Minos, Rhadamanthys und Sarpedon mythische Repräsentationsfiguren einer späteren machtvollen Dynastie auf Kreta. Die Geschichten, die sich um sie ranken, sind ein bedeutsamer Reflex griechischer Rückerinnerung an eine legendäre Zeit, in der Kreta die größte Macht in der Ägäis war. ${ }^{3} 8$

Freilich variiert auch diese Rückerinnerung nach Zeitstellung und soziokultureller Verankerung der Autoren; die Zusammenschau der Testimonien ergibt daher ein höchst widersprüchliches Bild. Dies gilt insbesondere für Minos, den prominentesten Spross der Vereinigung Zeus' und Europas. Der Herrscher von Knossos, nach dem die minoisch genannte Hochkultur Kretas (3000-I450 v. Chr.) benannt ist, hatte - nach Thuk. I,4 als erster - eine große Flotte, mit deren Hilfe er über eine ausgedehnte Seeherrschaft verfügte (vgl. auch Hdt. 3,I22), Seeräuber bekämpfte und viele Siedlungen gründete, u.a. auf den Kykladen (Diod. 5,84,I), wo er seine Nachkommen als Herrscher einsetzte. Von Europas drei Söhnen kommt ihm bei den Griechen nicht nur wegen seiner Herrschaft zu Wasser und zu Lande, seiner Gründeraktivität und weiträumigen Kommunikation das überwiegende Interesse zu, sondern auch infolge seiner innigen Beziehung zum obersten Griechengott selbst, der ihn persönlich erzog, sowie infolge der hieraus resultierenden Tätigkeit: Minos wurde Richter und erster Gesetzgeber Kretas bzw. der Menschheit überhaupt und damit auch der Nomothet der Griechen (Ps.-Plat. Min. 318c-e). Er erhielt, so wollten die Griechen wissen, seine Ausbildung als Richter und seine Gesetze vom Göttervater bei hierzu regelmäßig gepflegten Unterredungen in einer Höhle (Plat. leg. I,624a; Diod. $5,78,3)$. Dass es in Homers Odyssee heißt, er habe als Richter auch posthum in der Unterwelt gewirkt (Hom. Od. II,568-57I), hing damit zusammen, dass sein Ruf in dieser Tätigkeit außerordentlich gut war. In früher epischer Darstellung kam Minos als inniger Vertrauter und Sprachrohr des Zeus sehr viel besser weg als etwa in der attischen Tragödie, die ihn, da er der vermeintliche Besieger Attikas war, in ungünstigem Licht zeigte, während der eigene Großheros Theseus, der attischen Darstellung nach gegen den grausamen, un-

37 Zur Entwicklung des Inachidenstemmas (der Flussgott Inachos galt als Vater der Io; damit ist Io autochthone Argiverin) s. vor allem West I985, I45-I52; Yalouris I990, insbes. 663f.; Gantz I993, 202.

38 Vgl. Chaniotis 2004, I7-35. 
gerechten, tributheischenden Minos vorging, in hellem Licht erstrahlte. 39 Die Diskrepanzen in der Darstellung ein- und derselben Person fielen schon antiken Autoren auf, so dass geschlossen wurde, es habe zwei Herrscher mit Namen Minos gegeben (Diod. 4,60,3). Für die nachklassische Zeit bis hin zu Diodor lässt sich insgesamt beobachten, dass wieder ein eher positives Bild gezeichnet wird, das auf die nomothetische und politikbegründende Leistung des Zeus abhebt, die sich in Minos, der seines Vaters in Gerechtigkeit und Tapferkeit würdig ist, reproduziert und konkretisiert. $4{ }^{\circ}$

Als Minos nachgeordnet erscheint in der Überlieferung Rhadamanthys, der seit der Ilias (Hom. Il. I4,322) regelmäßig als Bruder von jenem geführt wird (z. B. Ps.-Plat. Min. 3I8d; Lukian. de luctu 7). Rhadamanthys gilt ebenfalls als Gesetzgeber auf Kreta sowie als Herrscher über die ägäischen Inseln und scheint überdies in Mittelgriechenland - in Boiotien war er als Gemahl der Alkmene bekannt (s.o.) - recht bedeutsam gewesen zu sein. Ihm wurden wichtige Rechtsgrundlagen für das griechische Prozesswesen zugeschrieben (Plat. leg. I, 625a), u.a. der Eid beider Parteien, an den der Richterspruch zu binden sei (Plat. leg. 12,948b-c).4 Auch das ius talionis, das Wiedervergeltungsrecht, und die Sitte, nicht bei Götternamen, sondern Tieren zu schwören, wurden auf ihn zurückgeführt (Aristot. eth. Nic. II32b 25f.; Porph. de abstinentia 3,I6). Rhadamanthys galt, im Vergleich zu seinem älteren Bruder, weitaus weniger als Machtmensch und wurde daher auch seltener negativ gezeichnet. Das gilt auch für den dritten Bruder, Sarpedon. Er soll nach Herodot, der angibt, die Lykier stammten ursprünglich aus Kreta, infolge eines Thronstreites mit Minos aus Kreta vertrieben worden und König der Lykier geworden sein (Hdt. I,I73). Andere Quellen nennen Sarpedon als Gründer von Milet (z.B. Ephor. fr. I27); in jedem Fall war auch er ein mobiler Heros. Nach Diodor $(5,79,3)$ war er der Großvater des Kämpfers Sarpedon, der den Griechen durch besonders tapfere Teilnahme am Trojanischen Krieg positiv auffiel. $4^{2}$

Es ließen sich an dieser Stelle sehr viel weitergehende Überlegungen zu sowohl den Söhnen als auch anderen Verwandten Europas anstellen. Vieles konnte hier nur angeschnitten werden und wäre zu entwickeln, um zu zeigen, wie komplexe Prozesse der Rezeption und Translation dazu führten, dass Wanderungen durch den Mittelmeerraum, wie von der nach Kreta verbrachten Europa paradigmatisch ,verkörpert‘, zu Gedächtnisfiguren wurden, die noch die heutige europäische Kultur prägen. Doch ist der zur Verfügung stehende Raum erschöpft. Die knapp angeführten Beispiele müssen genügen, um zu verdeut-

39 Für Überblicksdarstellungen zu Minos s. z. B. Hoeck I828, 45-56; I8I-2I4; Helbig I894-I897; Poland I932; Bažant i992.

40 Vgl. dazu Gehrke I997, 6I-64.

4I Für Überblicksdarstellungen zu Rhadamanthys s. z. B. Hoeck I828, 45-56; I8I-2I4; Jessen I9o9-I9I5; Xagorari 1994 .

42 Diodor versuchte so, mythenpragmatisch das von vielen Autoren bemerkte chronologische Problem zu lösen, dass der vor Troja kämpfende Sarpedon nicht in Minos' Zeit gehören konnte. - Für Überblicksdarstellungen zu Sarpedon s. z. B. Hoeck I828, 45-56; I8I-2I4; Immisch I909-I9I5; von Bothmer I994. 
lichen, wie vielfältige Anknüpfungs- und Legitimationsmöglichkeiten die genealogisch vernetzten Migrationsfiguren für verschiedene Formen einer Erinnerungspflege, die auf große zeitliche Tiefe setzte, boten. Mittels genealogischer Darstellung wurde vermeintliche Historie auf gekonnte Weise narrativ zur Schau gestellt. Geschichten von Zeus und den verschiedenen Heroinen und Heroen wurden zuhauf erzählt und nicht zuletzt in den Aktionsfeldern von Heiligtümern, Tempeln, Kultbildern und Ritualen auf verschiedenste Weise memoriert, indem ältere Informationen aufgegriffen und für eigene Aussagen über bestimmte Lokalitäten und Gruppen abgeändert wurden. Eines der Hauptprinzipien war es dabei, um ein vielzitiertes Wort Klaus Heinrichs zu verwenden, „die Macht der heiligen Ursprünge“ fächerartig „auf das von ihnen Abstammende, aus ihnen Abgeleitete“ zu übertragen. 43

Diese religiöse Komponente bei der narrativen Erinnerungspflege sei hier abschließend noch einmal herausgestellt: Am Anfang des Erzählens der griechischen Mythen, die um die Geschichte der Entführung Europas gruppiert sind und zu deren Prominenz nicht unwesentlich beigetragen haben dürften, steht der hieros gamos, der sich als in vielerlei Hinsicht fruchtbar erwies: Die Vereinigung des Zeus mit den Migrationsheroinen Io und Europa brachte, so erzählten die griechischen Dichter, jeweils ein neues Geschlecht hervor. Dieses Geschlecht war, wie oben exemplarisch an der Minos-Generation gezeigt, nicht nur aufgrund seiner bloßen Abstammung vom griechischen Göttervater bedeutend. Es war auch deshalb von substantieller Wichtigkeit, weil sich Zeus mit seinen Qualitäten in ihm reproduzierte und konkretisierte und weil es, wie für bestimmte Eliten in früher Zeit tatsächlich typisch, eine außerordentlich hohe Mobilität aufwies. Durch diese Mobilität wurde der ,heilige Samen ' des obersten Griechengottes in seinen qualitativen Konkretisierungen gleichsam durch den Mittelmeerraum getragen und in ihm verstreut.

Anders formuliert: Die mobilen Heroinen, mit denen sich Zeus verband, und die Geschlechter, die hieraus hervorgingen, spielen im Prozess der Besiedelungen, Auseinandersetzungen und Kulturbildungen im Mittelmeerraum eine wichtige Rolle und verweisen auf bedeutende Wurzeln griechischer bzw. von den Griechen adaptierter Geschichte und Kultur. Mit den Erzählungen hiervon definierten die Griechen ihre Ursprünge mythisch und verwiesen auf deren „heilige Macht“; zugleich erfuhren verschiedenste Prozesse im Mittelmeer- und Schwarzmeergebiet durch Übersetzung in griechische Geschichten eine Hellenisierung. Es wäre m. E. lohnend, umfänglich zu unternehmen, wozu dieser Artikel angeregt haben mag: Aus dem komplizierten Geflecht von Überlieferungen für die einzelnen Figuren und Generationen der Europafamilie zeit- und regionenspezifische ,Schichten' herauszupräparieren, um herauszufinden, wie die einzelnen Familienmitglieder im Kontext welches Lebensbereiches und welcher jeweiligen historisch-politischen Entwicklung zwecks Konstruktion von Wirklichkeit genealogisierend funktionalisiert wurden. 


\section{Literaturverzeichnis}

\section{Antonaccio 2007}

C. Antonaccio, „Colonization: Greece on the Move, 900-480“. In: H. A. Shapiro (Hrsg.), The Cambridge Companion to Archaic Greece, Cambridge 2007.

\section{Assmann 1988}

J. Assmann, „Kollektives Gedächtnis und kulturelle Identität“. In: J. Assmann/T. Hölscher (Hrsgg.), Kultur und Gedächtnis, Frankfurt a.M. I988, 9-19.

\section{Assmann 1997}

J. Assmann, Das kulturelle Gedächtnis. Schrift, Erinnerung und politische Identität in frühen Hochkulturen, München 1997.

\section{Bažant 1992}

J. Bažant, Art. „Minos I“. In: LIMC 6.I (I992), 570-574.

\section{Beekes 2004}

R. S. P. Beekes, „Kadmos and Europa, and the Phoenicians“. In: Kadmos (43) 2004, I67-I84.

\section{Berger/Luckmann 1966}

P. L. Berger/T. Luckmann, The Social Construction of Reality, New York I966; dt. Die gesellschaftliche Konstruktion der Wirklichkeit.

\section{von Bothmer 1994}

D. S. von Bothmer, Art. „Sarpedon“. In: LIMC 7.I (I994), 696-700.

\section{Bühler 1968}

W. Bühler, Europa. Ein Überblick über die Zeugnisse des Mythos in der antiken Literatur und Kunst, München ig68.

\section{Chaniotis 2004}

A. Chaniotis, Das antike Kreta, München 2004.

\section{Chaniotis 2006}

A. Chaniotis, „Die hellenistischen Kriege als Ursache von Migration: Das Beispiel Kreta“. In: E. Olshausen/H. Sonnabend (Hrsgg.), „Trojaner sind wir gewesen “- Migrationen in der antiken Welt. Stuttgarter Kolloquium zur Historischen Geographie des Altertums 8 (2006), Stuttgart 2006, 98-103.

\section{Cobet 1996}

J. Cobet, „Europa und Asien - Griechen und Barbaren - Osten und Westen. Zur Begründung Europas aus der Antike“. In: Geschichte in Wissenschaft und Unterricht (47/7-8) I996, 405-420.

\section{Coldstream 1993}

J. N. Coldstream, „Mixed Marriages at the Frontiers of the Early Greek World“. In: Oxford Journal of Archaeology (I2/I) I993, 80-I07. 


\section{Demandt 1998}

A. Demandt, „Europa: Begriff und Gedanke in der Antike“. In: P. Kneissl/V. Losemann (Hrsgg.), Imperium Romanum. Studien zu Geschichte und Rezeption. Festschrift für Karl Christ zum 75. Geburtstag, Stuttgart I998, I37-I57.

\section{Dombrowsky 1984}

B. W. W. Dombrowsky, Der Name Europas auf seinem griechischen und altsyrischen Hintergrund. Ein Beitrag zur ostmediterranen Kultur- und Religionsgeschichte in frühgriechischer Zeit, Amsterdam I984.

\section{Eitrem I9I6}

S. Eitrem, Art. „Io“. In: RE 9.2 (1916), Sp. I732-I743.

\section{Escher-Bürkli 1907}

J. Escher-Bürkli, Art. „Europe (I)“. In: RE 6.I (I907), Sp. I287-I298.

\section{Föllinger 2003}

S. Föllinger, Genosdependenzen. Studien zur Arbeit am Mythos bei Aischylos (Hypomnemata Bd. I48), Göttingen 2003.

\section{Gantz 1993}

T. Gantz, Early Greek Myth. A Guide to Literary and Artistic Sources, Baltimore/ London I993.

\section{Gehrke 1997}

H.-J. Gehrke, „Gewalt und Gesetz. Die soziale und politische Ordnung Kretas in der Archaischen und Klassischen Zeit“. In: Klio 79 (I997), 23-68.

\section{Gehrke 2000}

H.-J. Gehrke, „Ethnos, Phyle, Polis. Gemäßigt unorthodoxe Vermutungen“. In: P. FlenstedJensen/M. Herman Hansen/T. Heine Nielsen/L. Rubinstein (Hrsgg.), Polis and Politics: Studies in Ancient Greek History. Presented to Mogens Herman Hansen on his Sixtieth Birthday, August 20, 2000, Kopenhagen 2000, I59-I76.

\section{Gehrke 2003}

H.-J. Gehrke, „Was heißt und zu welchem Ende studiert man intentionale Geschichte? Marathon und Troja als fundierende Mythen“. In: G. Melville/K.-S. Rehberg (Hrsgg.), Gründungsmythen, Genealogien, Memorialzeichen. Beiträge zur institutionellen Konstruktion von Kontinuität, Köln u.a. 2003, 2I-36.

\section{Gehrke 2005}

H.-J. Gehrke, „Heroen als Grenzgänger zwischen Griechen und Barbaren“. In: E. S. Gruen (Hrsg.), Cultural Borrowings and Ethnic Appropriations in Antiquity, Stuttgart 2005, 50-67.

\section{Gehrke 2008}

H.-J. Gehrke, „Vergangenheitsrepräsentation bei den Griechen“. In: Internationales Jahrbuch für Hermeneutik 7 (2008), I-22.

Hall 1989

E. Hall, Inventing the Barbarian. Greek Self-Definition through Tragedy, Oxford I989. 
Hall 1997

J. M. Hall, Ethnic Identity in Greek Antiquity, Cambridge I997.

Hall 2002

J. M. Hall, Hellenicity. Between Ethnicity and Culture, Chicago/ London 2002.

\section{Hartmann 2003}

A. Hartmann, „Im Osten nicht Neues: Europa und seine Barbaren seit dem 5. Jahrhundert v. Chr.“. In: A. Michler/W. Schreiber (Hrsgg.), Blicke auf Europa. Kontinuität und Wandel, Neuried 2003, 3I-77.

\section{Heinrich 1966}

K. Heinrich, „Die Funktion der Genealogie im Mythos“. In: K. Heinrich, Parmenides und Jona. Vier Studien über das Verhältnis von Philosophie und Mythologie, Frankfurt a.M. I966, 9-28.

Helbig I884-I886

W. Helbig, Art. „Europa“. In: Roscher I (I884-I886), Sp. I409-I4I8.

Helbig I884-1886

W. Helbig, Art. „Minos“. In: Roscher 2.2 (1894-I897), Sp. 2993-3004.

Herman 1987

G. Herman, Ritualized Friendship and the Greek City, Cambridge u.a. I987.

\section{Hoeck 1828}

K. Hoeck, Kreta: Ein Versuch zur Aufhellung der Mythologie und Geschichte, der Religion und Verfassung dieser Insel, von den ältesten Zeiten bis auf die Römer-Herrschaft, Bd. 2, Göttingen I828.

Immisch 1909-1915

O. Immisch, Art. „Sarpedon“. In: Roscher 4 (1909-1915), Sp. 389-4I3.

Jessen 1909-1915

O. Jessen, Art. „Rhadamanthys“. In: Roscher 4 (1909-1915), Sp. 77-86.

\section{Kühr 2006}

A. Kühr, Als Kadmos nach Boiotien kam. Polis und Ethnos im Spiegel thebanischer Gründungsmythen, Stuttgart 2006.

\section{Kühr 2009}

A. Kühr, „Europa war nie in Europa. Mythos und Geographie in vorhellenistischer Zeit“. In: A.-B. Renger/R. A. Ißler (Hrsgg.): Europa - Stier und Sternenkranz. Von der Union mit Zeus zum Staatenverbund, Göttingen 2009, I03-II5.

\section{Miller 2005}

M. C. Miller, „Barbarian Lineage in Classical Greek Mythology and Art: Pelops, Danaos, Kadmos“. In: E. S. Gruen (Hrsg.), Cultural Borrowings and Ethnic Appropriations in Antiquity, Stuttgart 2005, 68-89.

\section{Mueller I89I}

M. Mueller, De Seleuco Homerico, Diss. Göttingen I89ı. 


\section{Musäus 2009}

I. Musäus, „Der Name Europas“. In: A.-B. Renger/R. A. Ißler (Hrsgg.), Europa - Stier und Sternenkranz. Von der Union mit Zeus zum Staatenverbund, Göttingen 2009, 34I-35I.

\section{Ninck 1945}

M. Ninck, Die Entdeckung von Europa durch die Griechen, Basel I945.

\section{Pfligersdorffer 1966}

G. Pfligersdorffer, Art. „Europa I (geographisch)“. In: RAC 6 (I966), Sp. 964-980.

\section{Poland 1932}

F. Poland, Art. „Minos“. In: RE 15.2 (I932), Sp. I890-1927.

\section{Prinz 1979}

F. Prinz, Gründungsmythen und Sagenchronologie, München I979.

\section{Raible 1998}

W. Raible, „Alterität und Identität“. In: Zeitschrift für Literaturwissenschaft und Linguistik IIo (I998), 7-22.

\section{Renger 2003}

A.-B. Renger (Hrsg.), Mythos Europa. Texte von Ovid bis Heiner Müller, Leipzig 2003.

\section{Renger/Ißler 2009}

A.-B. Renger/R. A. Ißler, „Stier und Sternenkranz: Europa in Mythos und Geschichte. Ein Rundgang“. In: A.-B. Renger/R. A. Ißler (Hrsgg.), Europa - Stier und Sternenkranz. Von der Union mit Zeus zum Staatenverbund, Göttingen 2009, 5I-99.

\section{Scheer 1993}

T. S. Scheer, Mythische Vorväter: Zur Bedeutung griechischer Heroenmythen im Selbstverständnis kleinasiatischer Städte, München I993.

\section{Schumacher 1990}

L. Schumacher, „Europa: Vom Mythos zur geographischen Vorstellung“. In: Ausst. Kat. Kreta. Das Erwachen Europas, Begleitband zur Ausstellung im Niederrheinischen Museum der Stadt Duisburg, 22. April bis 29. Juli I990, Athen u.a. I990, II-23.

\section{Weber 1972}

M. Weber, Wirtschaft und Gesellschaft. Grundriss der verstehenden Soziologie, 5. Aufl., Tübingen 1972 .

\section{West 1985}

M. L. West, The Hesiodic Catalogue of Women. Its Nature, Structure, and Origins, Oxford I985.

\section{Xagorari 1994}

M. Xagorari, Art. „Rhadamanthys“. In: LIMC 7.I (I994), 626-628.

\section{Yalouris 1990}

N. Yalouris, Art. „Io I“. In: LIMC 5.I (I990), 66I-676.

\section{Zahn 1983}

E. Zahn, Europa und der Stier, Würzburg I983. 